\title{
Simulation of Code Spectrum and Code Flow of Cultured Neuronal Networks
}

\author{
Shinichi Tamura, ${ }^{1}$ Yoshi Nishitani, ${ }^{2}$ Chie Hosokawa, ${ }^{3}$ \\ Tomomitsu Miyoshi, ${ }^{4}$ and Hajime Sawai ${ }^{5}$ \\ ${ }^{1}$ NBL Technovator Co., Ltd., 631 Shindachimakino, Sennan 590-0522, Japan \\ ${ }^{2}$ Department of Radiology, Graduate School of Medicine, Osaka University, Suita 565-0871, Japan \\ ${ }^{3}$ Biomedical Research Institute, AIST, Ikeda, Osaka 563-8577, Japan \\ ${ }^{4}$ Department of Integrative Physiology, Graduate School of Medicine, Osaka University, Suita 565-0871, Japan \\ ${ }^{5}$ College of Health and Human Sciences, Osaka Prefecture University, Habikino, Osaka 583-8555, Japan
}

Correspondence should be addressed to Shinichi Tamura; tamuras@nblmt.jp

Received 1 July 2015; Revised 1 October 2015; Accepted 25 October 2015

Academic Editor: Reinoud Maex

Copyright (c) 2016 Shinichi Tamura et al. This is an open access article distributed under the Creative Commons Attribution License, which permits unrestricted use, distribution, and reproduction in any medium, provided the original work is properly cited.

It has been shown that, in cultured neuronal networks on a multielectrode, pseudorandom-like sequences (codes) are detected, and they flow with some spatial decay constant. Each cultured neuronal network is characterized by a specific spectrum curve. That is, we may consider the spectrum curve as a "signature" of its associated neuronal network that is dependent on the characteristics of neurons and network configuration, including the weight distribution. In the present study, we used an integrate-and-fire model of neurons with intrinsic and instantaneous fluctuations of characteristics for performing a simulation of a code spectrum from multielectrodes on a 2D mesh neural network. We showed that it is possible to estimate the characteristics of neurons such as the distribution of number of neurons around each electrode and their refractory periods. Although this process is a reverse problem and theoretically the solutions are not sufficiently guaranteed, the parameters seem to be consistent with those of neurons. That is, the proposed neural network model may adequately reflect the behavior of a cultured neuronal network. Furthermore, such prospect is discussed that code analysis will provide a base of communication within a neural network that will also create a base of natural intelligence.

\section{Introduction}

Many generator models and analyzing methods of spike trains from neurons including artificial models, such as spikecoding metrics [1], spatiotemporal coding models [2-8], synchronous action models [9-13], and pseudorandom code detection methods [14] have been proposed.

On the other hand, we identified the sequence "1101" within the poststimulus time histogram (PSTH)/instantaneous firing rate (IFR) of a cultured neuronal network in Baljon et al. [15] (Figure 1) as well as our own network $[16,17]$ (Figure 2). The sequence "1101" is the core portion of the reversal M-sequence "1101000" which is a typical pseudorandom sequence [18-21]. Electric circuits generating pseudorandom sequences are well known. Furthermore, it has been shown that, in a cultured neuronal network on a multielectrode, pseudorandom-like sequences (codes), including "1101", are detected, and they flow with some spatial decay curve [22]. We call the chart of the appearance frequencies of short code (e.g., length $<8$ ) versus various codes (in number) "code spectrum." Each cultured neuronal network is characterized by a specific code spectrum curve. That is, the curve shape seems to depend on the characteristics of neurons and the network configuration, including the weight distribution in the network. Therefore, we may consider it as a "signature" of the network. In the present study, we performed a simulation of code spectrum from a multielectrode on a $2 \mathrm{D}$ mesh neural network using neurons with fluctuating characteristics of refractory period and output delay time around each given intrinsic characteristic from time to time; that is, parameters of each neuron varied intrinsically and temporarily. Connection weights between neurons are 


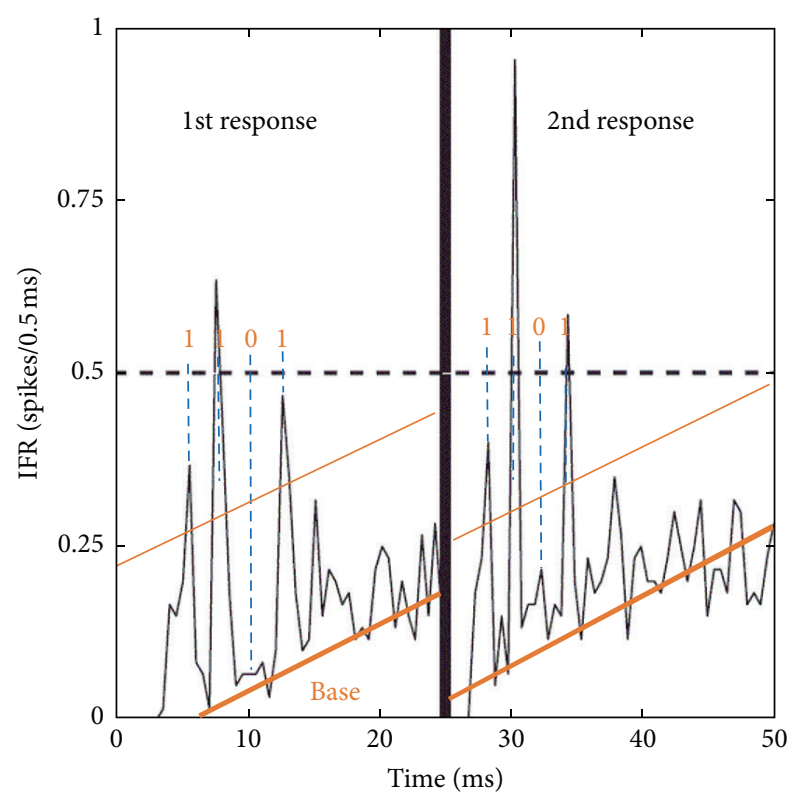

FIGURE 1: First and second responses "1101" in the instantaneous firing rate (IFR) of cultured neuronal tissue after two electrical stimulations as shown in [15] with additional interpretation (courtesy of Baljon et al.; reproduction permission provided by APS).

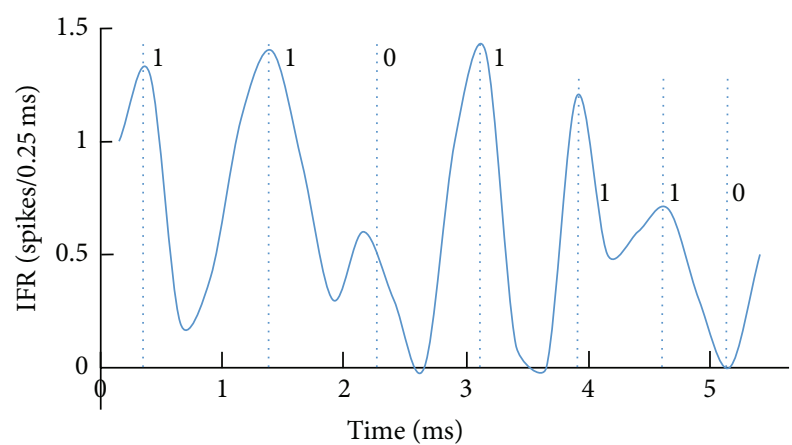

FIGURE 2: Instantaneous firing rate (poststimulus time histogram) where the sequence "1101" was observed. Since the timing becomes dispersed within trials, the peak positions and shapes gradually changed as time elapsed. Particularly, there was a tendency of risings and peaks to become faster than the regular timing, which is led by the fastest spike among the dispersed spikes as well as the slowest spike to be cancelled by the succeeding fastest negative spike (effectively supposed; pulling down the tail of peak), and lowering the peak height $[16,17]$.

randomly generated on the basis of a given statistical property. In this paper, expression "neuronal network" is mainly used for natural one, and "neural network" is for artificial one.

\section{Simulation}

2.1. Network Configuration. We performed a computer simulation to observe spike propagation on a $2 \mathrm{D}$ network with a $33 \times 332$ D mesh of neurons. Each neuron has connection

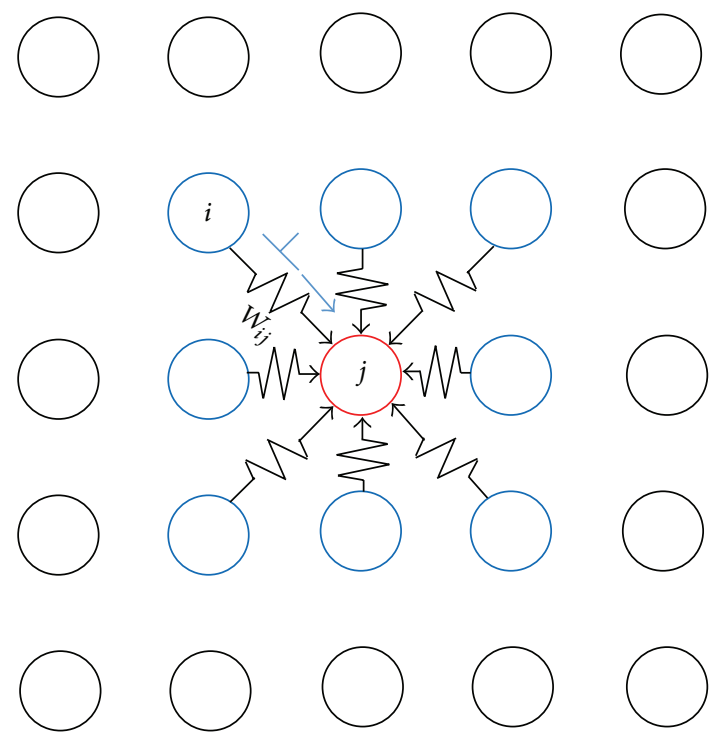

FIgURE 3: Inputs to neuron $j$ in a $2 \mathrm{D}$ mesh neural network. Each neuron $j$ (red) receives input spikes from eight neighboring neurons (blue) such as $i$ through connection weights $w_{i j} \in[-1,1]$. Neuron $j$ integrates such weighted spikes during its accepting period and outputs a spike after a delay time if the integrated value exceeds zero.

weights to and from eight neighboring neurons, as shown in Figure 3.

The weight of the network is given as follows:

$$
w_{i j}=F[(1+c) u-c], \quad w_{i j} \in[-1,1], w_{i j} \not \equiv w_{j i},
$$

where $i=\left(i_{x}, i_{y}\right) ; j=\left(j_{x}, j_{y}\right) ; i_{x}, i_{y}, j_{x}, j_{y} \in\{1,2, \ldots, 33\}$; $i \neq j ; \max \left\{\left|i_{x}-j_{x}\right|,\left|i_{y}-j_{y}\right|\right\}=1$; and $F[x]$ is a truncation function such that

$$
\begin{array}{rlrl}
F[x] & =1 & & 1 \leq x \\
& =x & & -1<x<1 \\
& =-1 & x \leq-1,
\end{array}
$$

$c$ is positive parameter such that $0<c \leq 3$ controls weight balance of positive and negative values and $u$ is a random variable with uniform distribution such that $0 \leq u<1$.

In short, if $c$ increases, the number of negative weights increases. For example, if $c=1 / 3$, weight $w_{i j}$ distributes uniformly in $[-1 / 3,1]$; that is, the ratio of positive to negative weights is $3: 1$. This ratio is a typical one in real neuronal networks. In $2 \mathrm{D}$ mesh artificial neural network model with weights to and from every eight neighboring neurons, however, weight distribution must be different because of different network shape and every eight neighboring weight is generated randomly, which usually does not result in zero. Of course, such models can be designed that first decide whether or not the weight exists and then decide on the weight value if it exists. For simplicity, we have designed that no connections between adjacent neurons (i.e., not connected; weight zero) are effectively realized by suppressions with negative weights. If $c=1, w_{i j}$ distributes in $[-1,1]$ uniformly; that is, the ratio of positive to negative weights is $1: 1$. If $c=3, w_{i j}$ distributes 


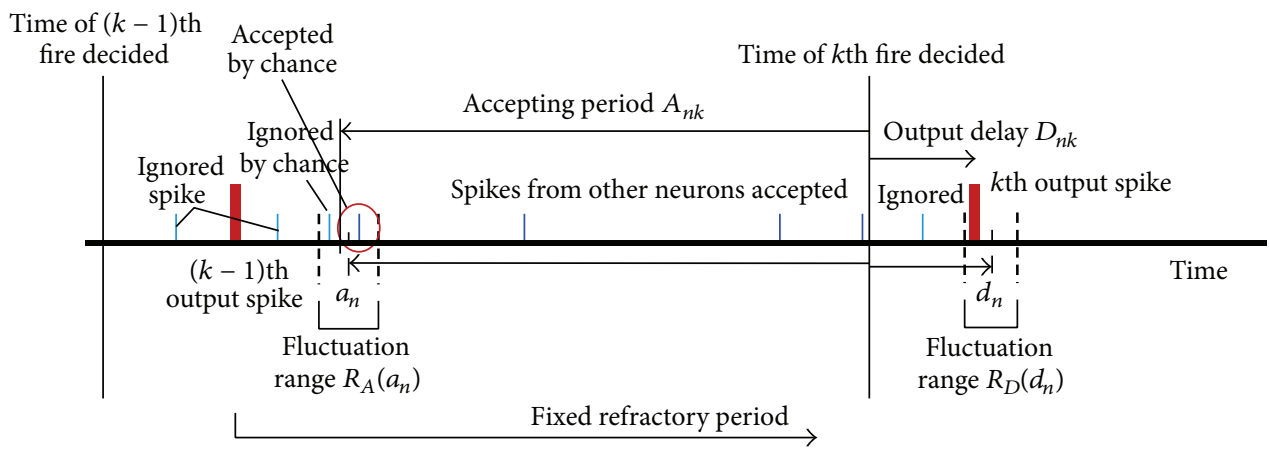

FIGURE 4: Integrate-and-fire model without leakage but with fluctuation in the parameters of neuron $n$. Each neuron has an inherent accepting period $a_{n}$ and output delay time $d_{n}$. These parameters vary with time within certain ranges $R_{A}\left(a_{n}\right)$ and $R_{D}\left(d_{n}\right)$, respectively. Neuron $n$ integrates weighted input spikes during the accepting period $A_{n k}$ for the $k$ th firing, and after the refractory period ends, it decides whether the integrated value exceeds zero for firing at every time point. If so, it outputs the $k$ th output spike with delay time $D_{n k}$. That is, $A_{n k} \in R_{A}\left(a_{n}\right)$, and likewise, $D_{n k} \in R_{D}\left(d_{n}\right)$.

in $[-1,1]$ but not uniformly; that is, the ratio of positive to negative weights is $1: 4$. Although the setting of weights was simple, the result was not much different from that obtained using separate settings of the weight values and their positive or negative signs.

2.2. Characteristics of Neurons. The neuron model used here is shown in Figure 4, which is a type of integrate-and-fire model without leak [25]. Neuron $n$ accumulates weighted inputs during the accepting period $A_{n k}$. If the accumulated weighted input becomes positive and the neuron is not in its refractory period, the neuron $n$ outputs a spike after a delay time $D_{n k}$ as the $k$ th firing. Some parameters used in the simulation are given as examples below.

A basic accepting period $a_{n}$ intrinsic to neuron $n$ is randomly generated in each network within a range $a_{n} \epsilon$ $\left\{a_{0}-2, a_{0}-1, a_{0}, a_{0}+1, a_{0}+2\right\}$ with a probability of $1 / 5$ for each, where $a_{0}$ is a fixed common parameter value through the network, and the unit time (bin) is $0.1 \mathrm{~ms}$. The actual instantaneous accepting period $A_{n k}$ of neuron $n$ at the $k$ th firing is further randomly given within the fluctuation range $R_{A}\left(a_{n}\right)$ around $a_{n}$; that is, $A_{n k} \in R_{A}\left(a_{n}\right)$. The instantaneous refractory period is implicitly assumed as slightly smaller than $A_{n k}$. The accepting period can also be called input integration or the accumulating period for firing. Roughly speaking, the accepting and refractory periods can be regarded as the same. The software can stimulate neurons with arbitrary spatiotemporal patterns. Typically, a combination of three neurons is simultaneously stimulated such that the states of the neurons are set to "1" at time 1 (bin no. 1). The reason why we stimulated three neurons simultaneously instead of only one neuron is that, in wet experiments, many neurons around an electrode seem to be simultaneously stimulated by an electrical stimulation; and secondly, parallel stimulation to multiple neurons seems to promote stable propagation of excitation according to computer simulations.

Parameters used in the simulation are as follows.

Basic accepting period $a_{0}$ is a preassigned network parameter such as $20,30, \ldots, 100$, where unit time (bin width) is $0.1 \mathrm{~ms}$. Instantaneous accepting period $A_{n k} \in$ $R_{A}\left(a_{n}\right)=\left\{a_{n}-1, a_{n}, a_{n}+1\right\}$ is randomly selected at the $k$ th firing with the probability $p_{a}, 1-2 \times p_{a}$, and $p_{a}$, respectively, and $p_{a}=1 / 12$. This value defines how much the instantaneous accepting period fluctuates from time to time. If we increase $p_{a}$ or expand the range $R_{A}\left(a_{n}\right)$, the fidelity of communication or information flow decreases. The above value is determined by considering a trade-off between such considerations and attaining 99\% communication fidelity. Details will be presented in our coming paper.

Similarly, the output delay is as follows.

Basic output delay time $d_{n}$ is randomly selected from $\{2,3, \ldots, 8\}$ with a probability of $1 / 7$ each. Instantaneous output delay time $D_{n k} \in R_{D}\left(d_{n}\right)=\left\{d_{n}-1, d_{n}, d_{n}+1\right\}$, and $p_{d}=1 / 12$, similar to $R_{A}\left(a_{n}\right)$.

We have utilized an integrate-and-fire model without leaks, but instead we used a model incorporating a fluctuating period of acceptance. Our model is more stringent than the leaky integrate-and-fire model because our model randomly neglects old spikes, while the leaky model neglects them gradually. However, from our simulations, ignoring a portion of the spikes in any type is not fatal and not essential but only influences the quality of communication. This property is a strong point of neural networks that are composed of essentially fluctuating neurons. The fluctuating time parameters are concentrated in the two parameters-the accepting period, preceding the fire deciding time and the output delay time, following this time. These designs were determined by balancing the degree of complication, essential precision of results, and computation time.

Results of a preliminary experiment of stimulating a $9 \times$ 9 mesh neural network are shown in Figure 5, where three neurons were stimulated at time $t=0$. We can observe "spike waves" propagating from source stimulations, and the waves often behave like Synfire [9, 10]. Further, the code flow may be regarded as a component of such spike waves. It is possible to acquire codes in wet experiments and compare them with the simulation. In the present study, however, as a preliminary step, we focused on simulating not only the entire spike waves but also the flow of codes.

2.3. Arrangement of Multielectrode. Figure 6 shows the arrangement of a multielectrode on a $33 \times 332 \mathrm{D}$ mesh neural 


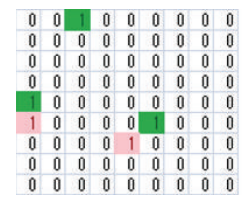

$0.1-0.5$

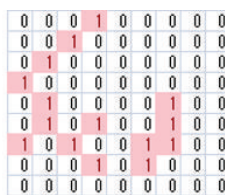

$0.6-1.0$

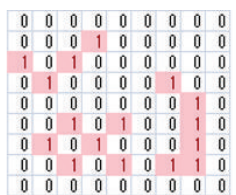

$1.1-1.5$

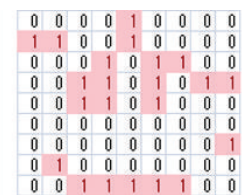

$1.6-2.0$

(ms)

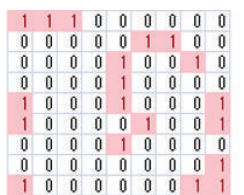

$2.1-2.5$

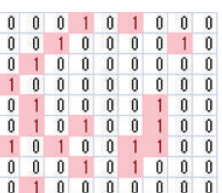

$2.6-3.0$

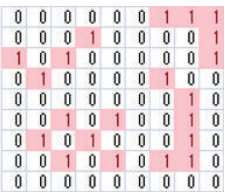

$3.1-3.5$

FIGURE 5: Spike waves generated on a 2D mesh neural network. Green spots indicate stimulation point. "0.1-0.5 ms" means an accumulation result of firing at $0.1 \mathrm{~ms}, 0.2 \mathrm{~ms}, \ldots, 0.5 \mathrm{~ms}$. This suggests that the codes are a part of these "spike waves."

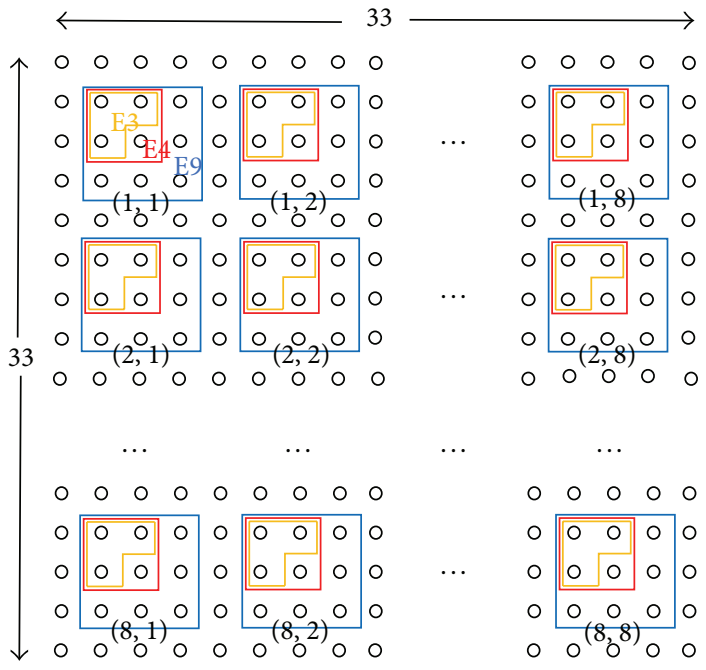

Figure 6: Arrangement of $8 \times 8$ multi-electrodes $[(1,1),(1,2), \ldots,(8,8)]$ on a simulated $33 \times 332 \mathrm{D}$ mesh neural network. Each electrode acquires spikes of two to nine neurons. For example, "E9" showing $3 \times 3$ block of neurons $(O)$ indicated with $(1,1)$ shows that electrode $(1,1)$ collects spikes from nine neurons. In addition, "E3" and "E4" are likewise; "E2" and "E5"-"E8" are not shown. Spectrum, cross-correlation, or probability distribution data obtained from electrode Em is expressed as " $E_{m}$ " in the text. Connections between eight neighboring neurons are randomly generated with given stochastic characteristics.

network. Each neuron has directional connections with eight neighboring neurons. Each electrode gathers spikes from two to nine surrounding neurons. The major differences from the wet experimental configuration [22] are as follows: (1) there are no neurons and no connections outside the explicit 1089 neurons, (2) neurons are placed on a regular lattice, which is different from an irregularly shaped cultured neuronal network, and (3) distances between electrodes are smaller than those in the cultured neuronal network. It can be said that difference (3) is a smaller scale of (1) and it corresponds to the fact that we regard the spike data from different electrodes as independent events. That is, in (1) and (3), we neglect the behavior of neurons outside the noted neurons.

2.4. Component Code Spectrum. By changing the basic accepting period $a_{0}$ and the positive and negative weight balance parameter $c$, we can generate spike trains for each type of electrode Em catching from $m(m=2,3, \ldots, 9)$ surrounding neurons. We then decode the spike trains to obtain code spectra for each $m$. We call such a spectrum $E_{m}$ "component spectrum." Figure 7 shows an example of simulated spike trains caught by electrode Em.

Figure 8 shows examples of component code spectra of simulated spike sequences of some $a_{0}$ (median of accepting period $A_{n k}$ ) and the connection weight parameter $c$. Practically, the number of neurons emitting spikes to each electrode changes electrode by electrode according to its statistical distribution. Therefore, the actual observed code spectra will be mixed according to the probability distribution of the number of neurons $m$ around the electrodes.

2.5. Spectrum Fitting with Components. The blue curve in Figure 9(a) shows the results of the average number of codes detected in recorded spike trains of 9 trials for code numbers 1-21 (code spectrum) from 63 electrodes of Sample A during $200 \mathrm{~ms}$ after stimulation expressed as 2000 times the bin data. Sample A is the same as that presented in [22].

The orange curve in Figure 9(a) shows the best fit to the number of codes detected in spike trains of 2000 time bins of Sample A using code spectra $E_{m}$ of artificially generated spike sequences with different accepting periods $a_{0}$ and connection weight parameters $c$ and probability distribution of $E_{m}$. Although this is an inverse problem and imperfect, the estimation of neuronal parameters $a_{0}, c$, and probability distribution of $E_{m}$ is possible to some extent.

Table 1 shows the normalized squared error of a simulated code spectrum to that of recorded data of Sample A with a changing probability distribution of $E_{m}$, that is, a combination of component code spectra for various $a_{0}$ and $c$ values. 


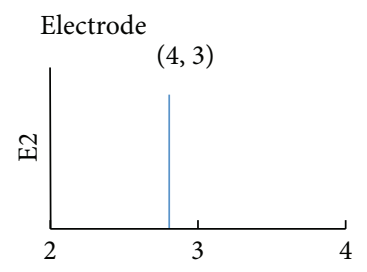

(ms)

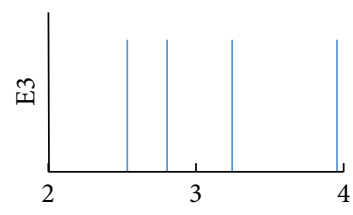

(ms)

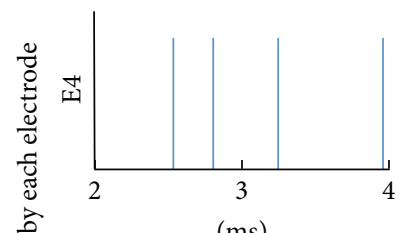

(ms)

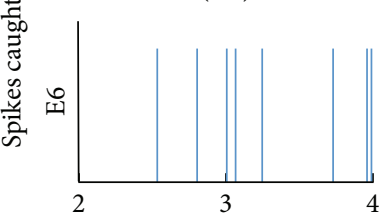

(ms)

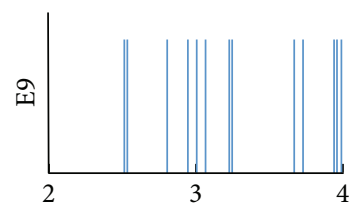

(ms)

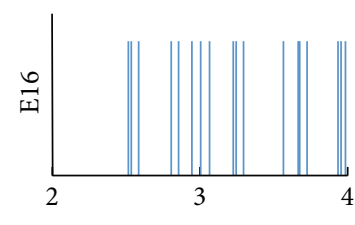

(ms)
$(4,4)$

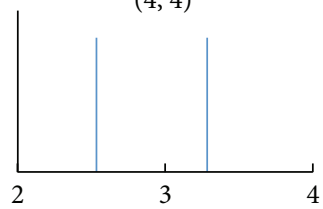

(ms)

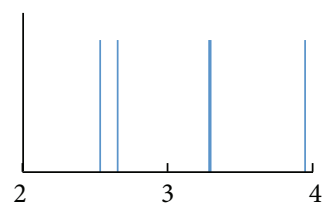

(ms)
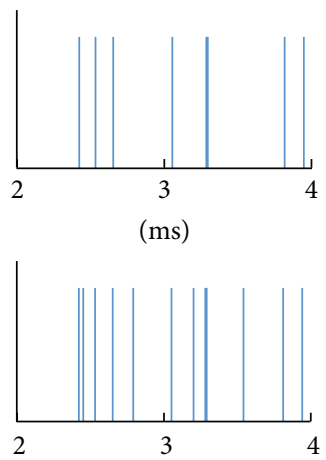

(ms)

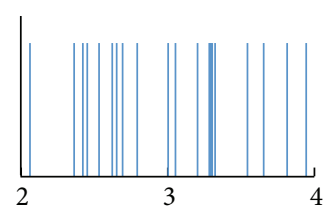

(ms)

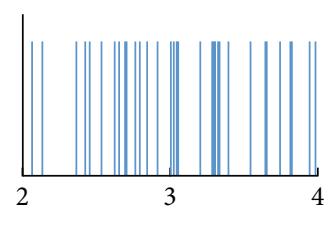

$(4,5)$

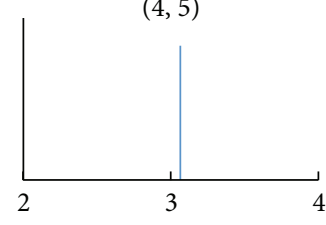

(ms)

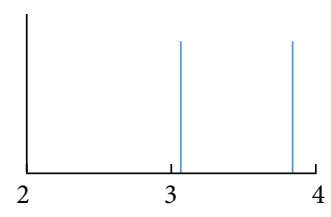

(ms)
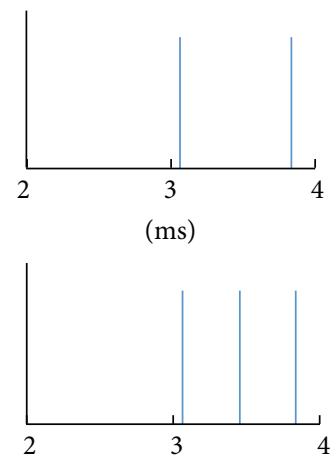

(ms)
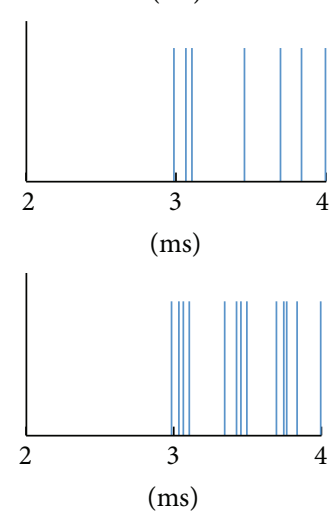

$(4,6)$
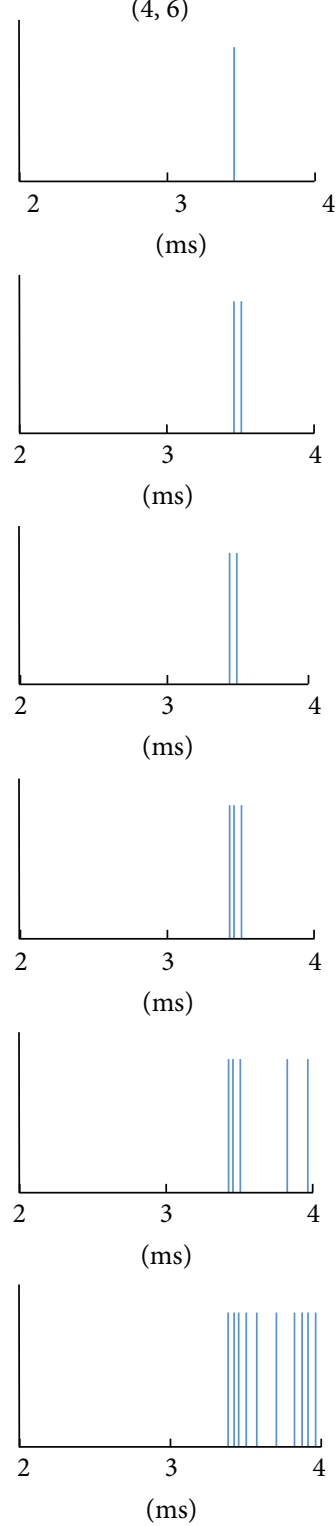

FIgure 7: An example of simulated spike trains caught by Em during 2.0-4.0 ms after stimulation with $a_{0}=8.0 \mathrm{~ms}$ and $c=2.5$. Initial stimulations were given to around electrodes $(1,1)$ at $0.1 \mathrm{~ms}$ and $(1,5)$ at $0.5 \mathrm{~ms}$.

Some root mean square (RMS) error data are not shown (shown as "-_") because the number of codes detected was too small to calculate the component code spectrum as such spikes disappeared within the given time. Tables 2 and 3 show that of Sample B in Figure 9(b) and Sample C in Figure 9(c), respectively.

2.6. Expansion of $E_{m}$. In some cases, the best estimation of the probability distribution of $E_{m}$ had a large value at $E_{9}$, suggesting that there are more than nine neurons around the electrode. Therefore, we increased the number $m$ to 16 . Figure 10 shows the expanded code spectral components up to $E_{16}$. Although $E_{2}-E_{9}$ have various shapes, $E_{9}-E_{16}$ have a similar shape. When the best fit process included a large number of parameters, the computation time was long.
Therefore, it may be reasonable to regard $E_{9}$ as the representative component of $E_{9}-E_{16}$ to decrease the computation time.

2.7. Maximum Cross-Correlations. Because the simulation size was limited, the separation distances between the electrodes were very small (1-4 times of the neuron pitch). Nevertheless, using the same method as used in [22] for the natural neuronal network, we calculated the maximum crosscorrelation $\Phi_{N}(C)$ of a trial among eight and 20 neighbors between a time frame difference of $0.5(\mathrm{~ms})$ for $E_{2}-E_{16}$ and 14 major codes $C$ (Figure 11). However, since the electrodes were located relatively closer than in [22], and therefore cooccurrence probability of each code between two electrodes was expected higher, normalization of cross-correlation by code 

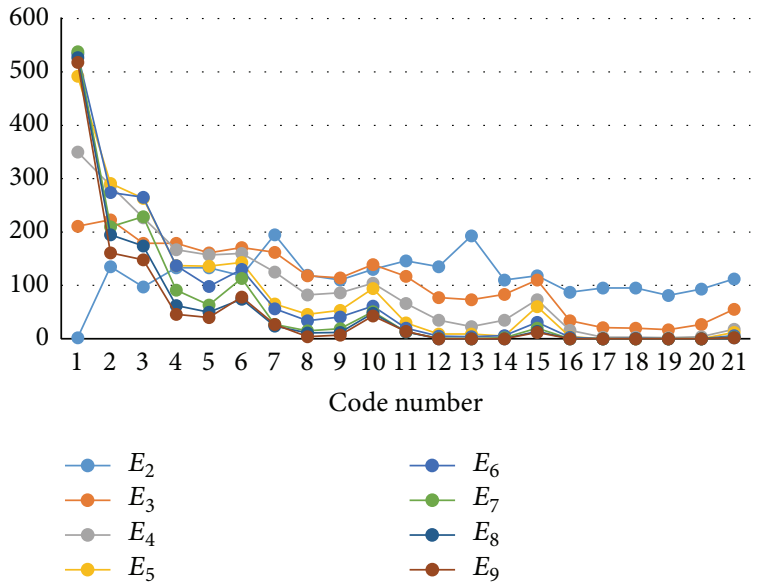

(a) $a_{0}=6 \mathrm{~ms} c=0.1$

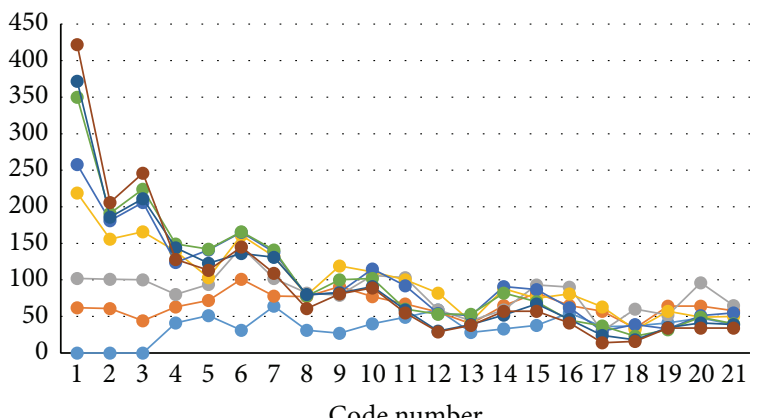

Code number

$\begin{array}{ll}\multimap E_{2} & \multimap E_{6} \\ \multimap E_{3} & \multimap E_{7} \\ \multimap E_{4} & \multimap E_{8} \\ \multimap E_{5} & \multimap E_{9}\end{array}$

(c) $a_{0}=9 \mathrm{~ms} c=1$
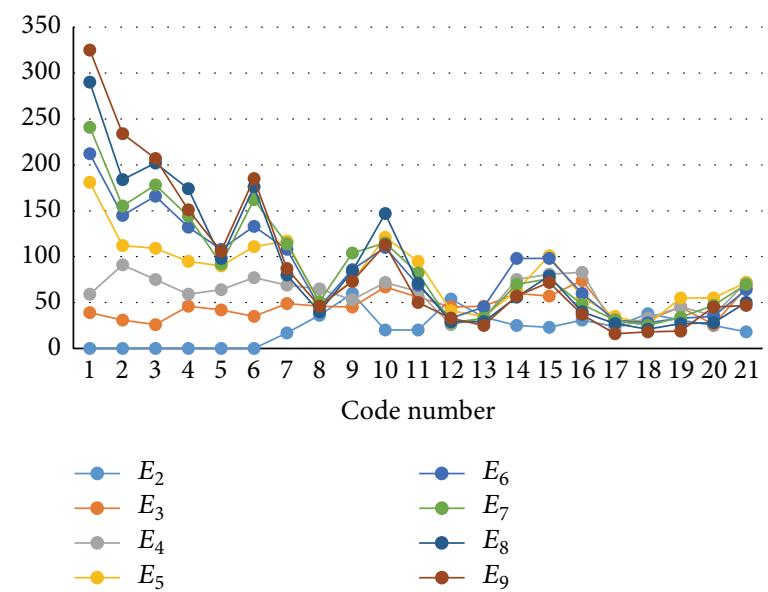

(e) $a_{0}=10 \mathrm{~ms} c=1$

FIGURE 8: Code spectrum components $E_{m}$ for several parameters of accepting period $a_{0}$ and positive and negative weight balance $c$. The horizontal line represents code numbers $(1,2, \ldots, 21)$ whose number of "1"s in the code is 3 . That is, code $1=$ " 111 ," code $2=$ " 1011 ," code $3=$ "1101," code $4=$ "10011," code $5=$ "10101," . . code $21=$ "11000001" [22]. The vertical line represents the total number of codes detected during the first $200 \mathrm{~ms}$ after stimulation (2000 time bins of $0.1 \mathrm{~ms} / \mathrm{bin}$ ) and from 63 electrodes. 


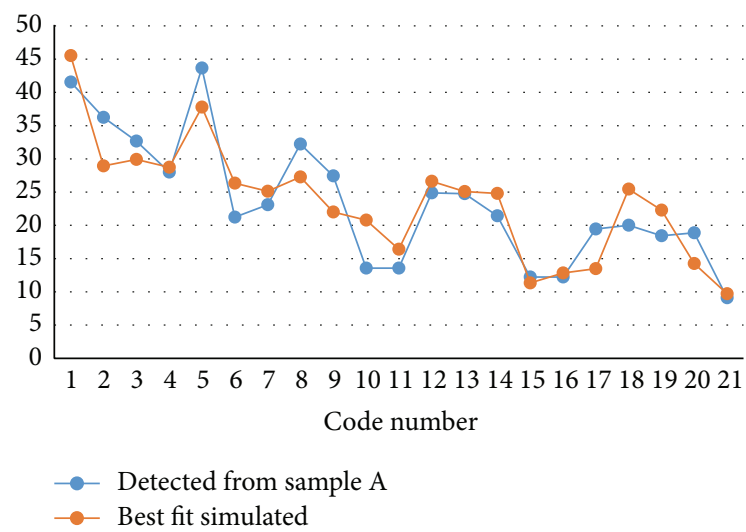

(a) Sample A ( 9 trials). Estimated $a_{0}=8 \mathrm{~ms}, c=2.5$, and probability distribution of $E_{m}(m=2,3, \ldots, 9)$ is $(0.3,0.1,0,0,0,0.05,0$, $0.55)$, with a normalized RMS error of 0.179

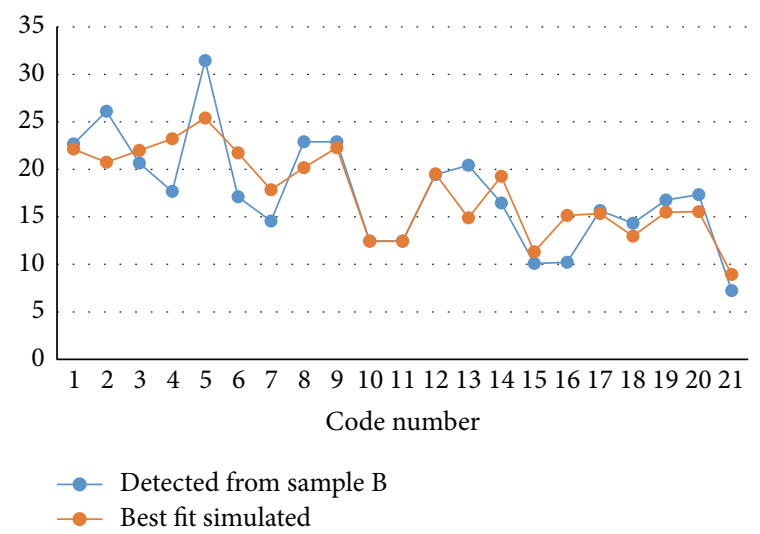

(b) Sample B (23 trials). Estimated $a_{0}=9 \mathrm{~ms}, c=2$, and probability distribution of $E_{m}(m=2,3, \ldots, 9)$ is $(0.3,0.1,0,0,0,0.05,0,0.55)$, with a normalized RMS error of 0.181

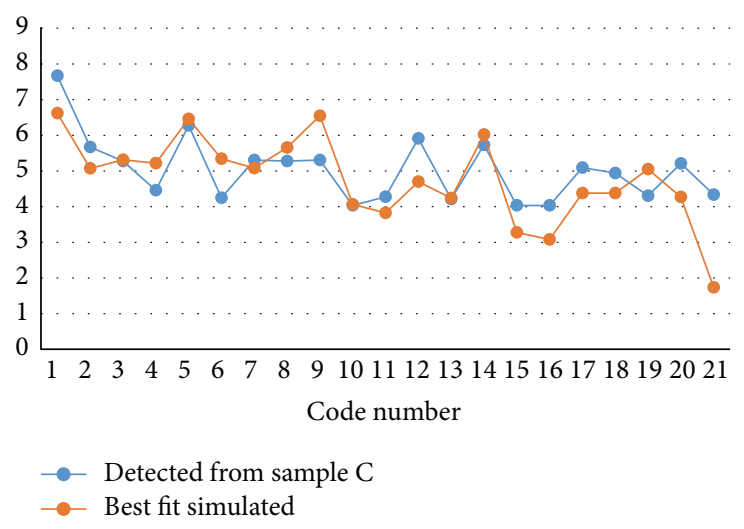

(c) Sample C (26 trials). Estimated $a_{0}=9 \mathrm{~ms}, c=2.5$, and probability distribution of $E_{m}(m=2,3, \ldots, 9)$ is $(0.263,0.158$, $0,0.053,0.053,0.053,0.421,0)$, with a normalized RMS error of 0.180

FIGURE 9: Code spectra. The blue curve shows the average of trials of an experimental number of codes detected from 63 electrodes in spike trains during $200 \mathrm{~ms}$ after stimulation expressed with 2000 time bins of $0.1 \mathrm{~ms}$. The bit width of the code is $0.6-2.0 \mathrm{~ms}$ (6-20 bins). Codes are detected with $1 \%$ time accuracy, although practically several \% because of the $0.1 \mathrm{~ms}$ bit width. This curve can be considered as the "signature" of spike trains. The orange curve is the best fit to code spectrum using a simulation spectrum.

TABLE 1: Normalized RMS error for various parameters of neurons used to fit the data in Figure 9(a). Bold and italic values show the first and second best fits, respectively. Typical $a_{0}$ and $c$ values were estimated as $8.0 \mathrm{~ms}$ and 2.5 , respectively.

\begin{tabular}{lcccccc}
\hline$c$ & \multicolumn{7}{c}{$a_{0}(\mathrm{~ms})$} \\
& 5.5 & 6.0 & 7.0 & 8.0 & 9.0 & 10.0 \\
\hline 0.1 & 0.464 & 0.315 & 0.565 & - & - & - \\
0.3 & 0.442 & 0.283 & 0.581 & 0.904 & - & - \\
1.0 & 0.393 & 0.252 & 0.182 & 0.212 & 0.327 & 0.349 \\
1.5 & 0.326 & 0.240 & 0.208 & 0.230 & 0.269 & 0.286 \\
2.0 & 0.301 & 0.246 & 0.203 & 0.212 & 0.186 & 0.310 \\
2.5 & - & 0.470 & 0.580 & $\mathbf{0 . 1 7 9}$ & 0.192 & - \\
3.0 & - & 0.321 & - & 0.328 & 0.319 & 0.674 \\
\hline
\end{tabular}

length was not applied. As shown in Figure 11(a), in case of $E_{4}$ and $E_{5}$, the maximum cross-correlation is rather flat in comparison with that of Sample A of the natural neuronal
TABLE 2: Normalized RMS errors for various parameters of neurons used to fit the data in Figure 9(b).

\begin{tabular}{lcccccc}
\hline$c$ & \multicolumn{6}{c}{$a_{0}(\mathrm{~ms})$} \\
& 5.5 & 6.0 & 7.0 & 8.0 & 9.0 & 10.0 \\
\hline 0.1 & 0.502 & 0.322 & 0.577 & - & - & - \\
0.3 & 0.484 & 0.288 & 0.577 & 0.890 & - & - \\
1.0 & 0.438 & 0.259 & 0.197 & 0.233 & 0.335 & 0.340 \\
1.5 & 0.361 & 0.243 & 0.210 & 0.248 & 0.271 & 0.283 \\
2.0 & 0.320 & 0.256 & 0.188 & 0.241 & $\mathbf{0 . 1 8 0 9}$ & 0.304 \\
2.5 & - & 0.477 & 0.605 & 0.203 & 0.1814 & - \\
3.0 & - & 0.325 & - & 0.317 & 0.332 & 0.680 \\
\hline
\end{tabular}

network of [22]. The characteristics of $E_{6}-E_{11}$ (Figure 11(b)) decreased according to the code number, which roughly represents the code length. Although not shown in figures, $E_{12}-E_{16}$ showed a more steep decreasing tendency. 

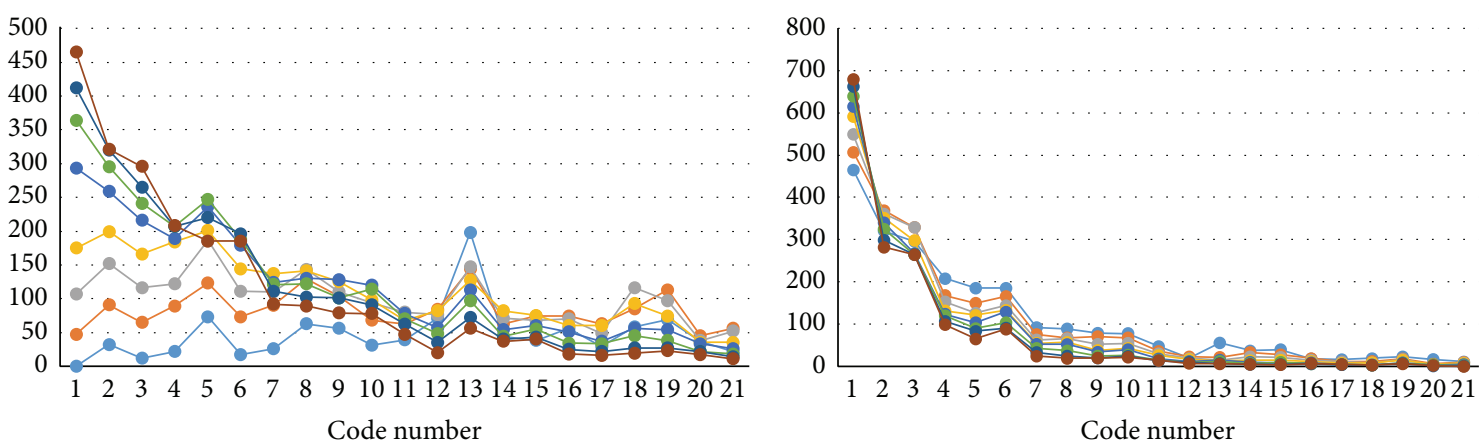

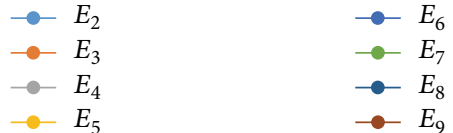

(a) $a_{0}=7 \mathrm{~ms} c=2 E_{2-9}$

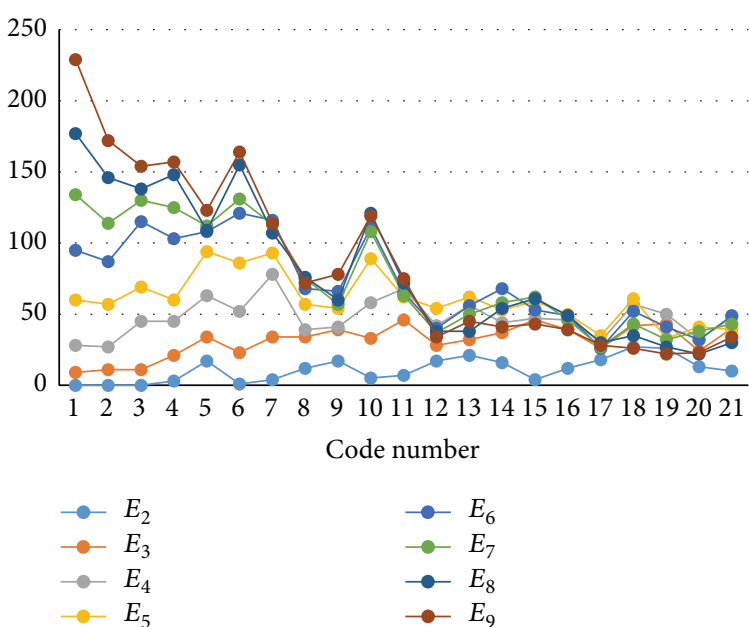

(c) $a_{0}=8 \mathrm{~ms} c=2.5 E_{2-9}$

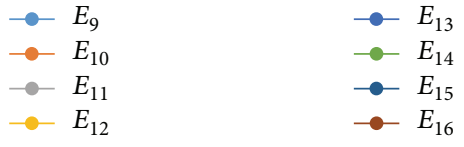

(b) $a_{0}=7 \mathrm{~ms} c=2 E_{9-16}$

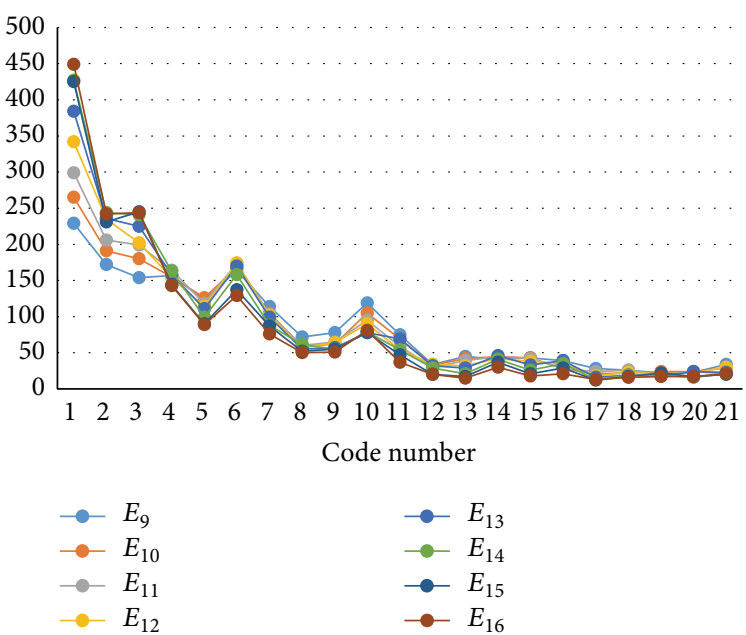

(d) $a_{0}=8 \mathrm{~ms} c=2.5 E_{9-16}$

Figure 10: Expanded spectrum components up to $E_{16}$. (a) Code spectrum components $E_{2}-E_{9}$ for $a_{0}=7 \mathrm{~ms}$ and $c=2$. (b) Expansion to $E_{9}-E_{16}$ of (a). (c) Code spectrum components $E_{2}-E_{9}$ for $a_{0}=8 \mathrm{~ms}$ and $c=2.5$. (d) Expansion to $E_{9}-E_{16}$ of (c).

TABLE 3: Normalized RMS errors for various parameters of neuron used to fit the data in Figure 9(c).

\begin{tabular}{lcccccc}
\hline$c$ & \multicolumn{6}{c}{$a_{0}(\mathrm{~ms})$} \\
& 5.5 & 6.0 & 7.0 & 8.0 & 9.0 & 10.0 \\
\hline 0.1 & 0.518 & 0.290 & 0.461 & - & - & - \\
0.3 & 0.509 & 0.223 & 0.440 & 0.777 & - & - \\
1.0 & 0.515 & 0.291 & 0.252 & 0.204 & 0.222 & 0.223 \\
1.5 & 0.430 & 0.275 & 0.253 & 0.196 & 0.197 & 0.187 \\
2.0 & 0.399 & 0.257 & 0.263 & 0.256 & 0.186 & 0.215 \\
2.5 & - & 0.491 & 0.656 & 0.213 & $\mathbf{0 . 1 8 0}$ & - \\
3.0 & - & 0.324 & - & 0.354 & 0.358 & 0.631 \\
\hline
\end{tabular}

\section{Discussion and Conclusions}

In the present study, we constructed a $2 \mathrm{D}$ mesh neural network model and an input/output (I/O) functional model of neurons. A 2D mesh model may differ from a real natural cultured neuronal network, which will have a nonhomogeneous distribution of neurons [26]. The I/O functional model of neurons has certain intrinsic and instantaneous fluctuations of the refractory period and output delay time [11]. There may be some discrepancies compared with real natural cultured neurons; however, we have shown that from the perspective of the code spectrum of spike trains, it is possible to simulate the electrical activity of a neuronal network on a multielectrode plate (MED plate) with a 2D mesh neural network model. Moreover, it was possible to estimate the neuronal network parameters to some extent by recording spike trains with electrode without sorting. For example, though there is no guarantee to be true ones, estimated typical accepting periods were $8-9 \mathrm{~ms}$, and refractory periods were also estimated as almost the same which are within range $(5-10 \mathrm{~ms})$ of a text book [27].

The detected codes were composed of spikes from different neurons because we acquired codes with bit widths ranging from 0.6 to $2.0 \mathrm{~ms}$; in cases of 4-bit code, code lengths are mainly less than $6 \mathrm{~ms}(=2 \mathrm{~ms} \times 3$ [spans of 4 -bit code] $)$. These values are generally shorter than the refractory period 


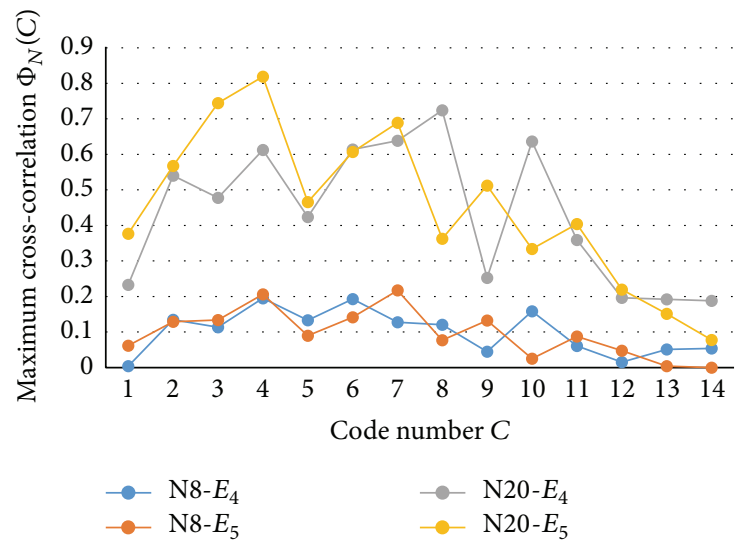

(a) $E_{4}-E_{5}$

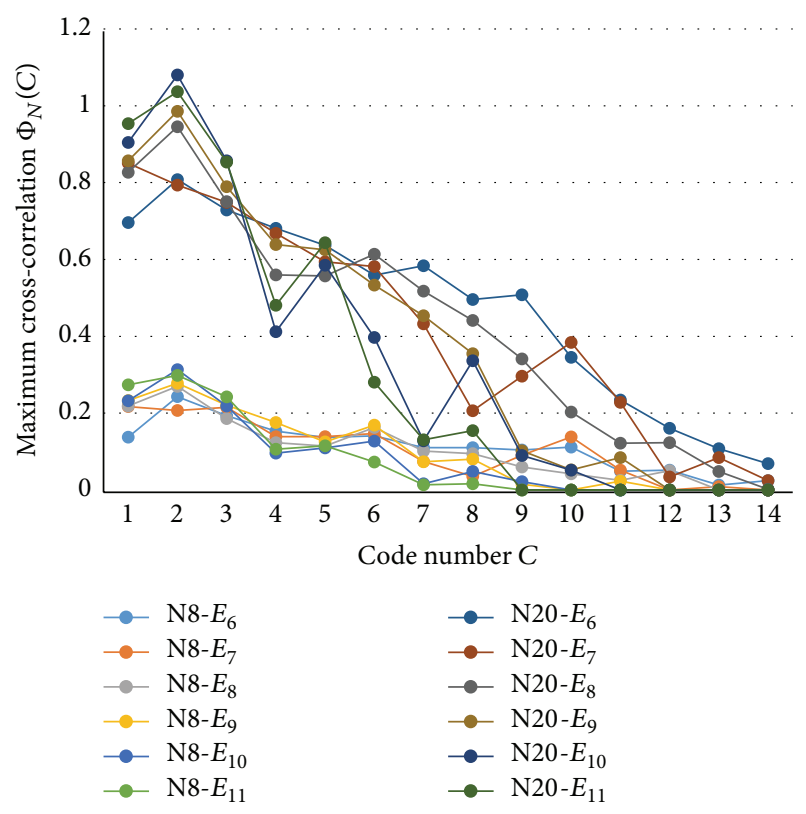

(b) $E_{6}-E_{11}$

FIGURE 11: Maximum cross-correlation $\Phi_{N}(C)$ of a trial among eight $(8 \mathrm{~N})$ and $20(20 \mathrm{~N})$ neighbors with a time frame difference of $0.5 \mathrm{~ms}$ for 14 major codes $C$.

of neurons, which is estimated to be more than $8 \mathrm{~ms}$ in our cases. This suggests that it is unnecessary to add sorting processing to the spikes obtained from each electrode in case of analyzing by code with length less than $8 \mathrm{~ms}$. That is, spikes forming the codes under our conditions originate from different neurons, and it may be possible to analyze circuit states around the electrodes. We need not to deal with $E_{1}$ whose spike interval is more than $8 \mathrm{~ms}$. In our case, although we statistically analyzed all the 63 electrodes (except for the stimulating electrode) together, it may be possible to analyze individual electrodes, which may give more precise distribution of $E_{m}$; that is, we can determine whether neurons near a certain electrode compose a specific circuit such as a linear feedback shift register (LFSR) [14].

We used simulated code spectrum components to analyze the wet experimental spectrum of a cultured neuronal network. It is true, however, there are problems of (1) besides codes are not always orthogonal, (2) some of the extracted codes may be false such that composed of overlapped spike combination by chance [22]. To reduce such effects, it may be effective to improve the statistical background such as increasing the number of spike trains to suppress the statistical variation of the code spectrum components, because the present data were acquired from only one spike train of one trial each, that is, from the 63 electrodes, which are not necessarily perfectly independent.

A 2D mesh neural network can generate spike waves, as shown in Figure 4. Thus, the code flow observed on a cultured neuronal network can be regarded as a fragment of "spike waves." Code not only works as a marker of the spike waves but also provides information or clues about the circuit's shape. In other words, the code spectrum reflects the circuit shape, including the weight distribution, neuron characteristics, and its role in communication around each electrode. Therefore, it may be considered as a kind of network signature.

Furthermore, spike waves will establish asynchronous multiplex communication links as well as multiplex communication within a synchronous neural network [23, 28] where various pseudorandom-like codes are observed. For communication within this network, each neuron or group of neurons cannot receive entire spike waves, but rather a specific part of the wave, that is, a pseudorandom-like code or a spatiotemporal combination of codes. Then, based on the communication links as presented in Figure 12 [24, 29], information will be processed by an intelligence mechanism in the brain. Under these conditions, information will be radiated as spike waves from source neurons and then widely propagated via the neuronal network. There has been substantial research on behavior of a spike intensity wave as a global macrotask including cardiac electric propagation [30-32]. Although it may be true that most neurons work to relay spikes as a part of spike waves, destination neurons in a communication task are limited in number and must select signals sent to them from among the various spike waves based on spike trains containing codes that are a fragment of each spike wave or a spatiotemporal combination of these codes. This task is local and should not be treated as conventional "intensity" level wave information, but rather as "locally phasic" level wave information incorporated in the spatiotemporal pattern of locally arriving spikes including codes. In artificial brain research, though through concentrated digital synaptic switching, effect of communication is shown [33]. The present study serves a base of communication tasks in natural 


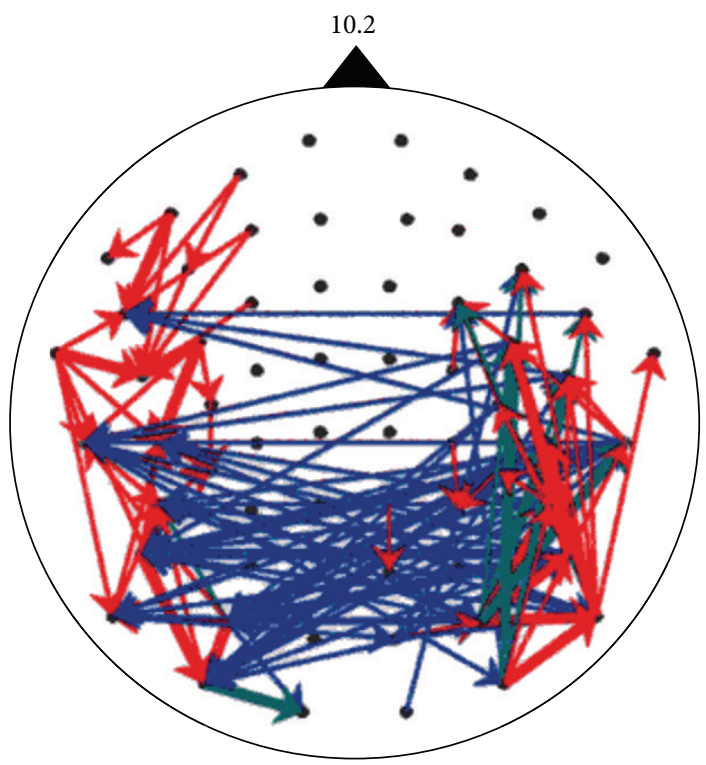

FIGURE 12: Time-shift diagram of $10.2 \mathrm{~Hz}$ MEG, for a number counting task [23, 24]. Red arrow $<5 \mathrm{~ms}<$ green $<10 \mathrm{~ms}<$ blue. We can see that red arrow with lag time $<5 \mathrm{~ms}$ runs within each hemisphere, and blue $>10 \mathrm{~ms}$ across the callosum.

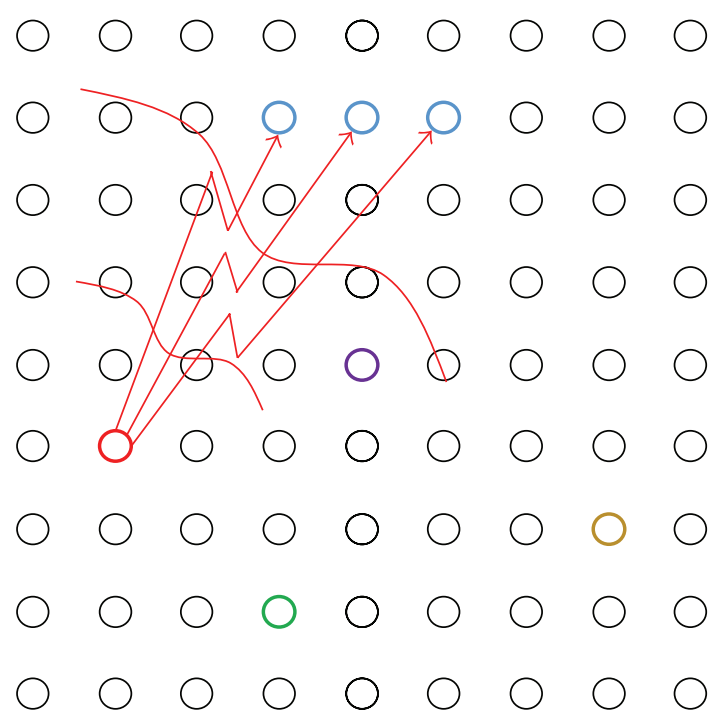

(a) The top three receiving neurons can detect the specific transmitting neuron among the four neurons from the spike wave fragment as a combination of spike codes (the spatiotemporal spike pattern)

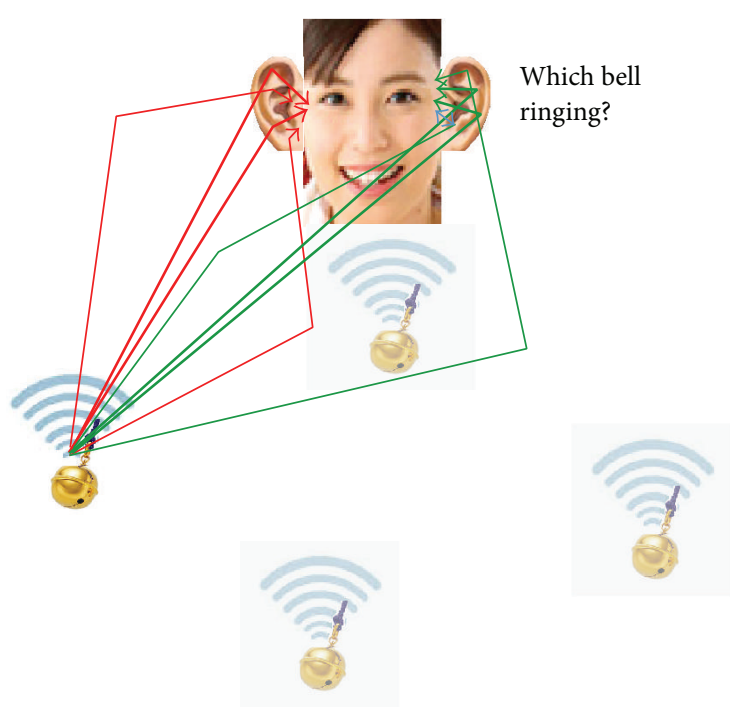

(b) The same mechanism allows us to perceive where a bell is ringing in our surrounding atmosphere

FIGURE 13: Illustration of the communication within the neural network based on spatiotemporal pattern recognition. Each neuron can perform the roles of transmitting, receiving, and simply as a transmission media.

intelligence, as illustrated in Figure 13. These studies of spike waves will lead to higher-order reasoning/intelligence in the brain via communication $[8,34]$.

Our results, including those reported in [22], are summarized as follows:

(1) We are investigating an intelligence mechanism with the maintenance of correspondence between wet experiments and simulation.

(2) We identified a pseudorandom sequence (code) "1101" for PSTH in the literature as well as from our experience which should provide clues about neuronal circuits around electrodes.

(3) A code spectrum as an extension of code "1101" was obtained from cultured neuronal networks and simulations.

(4) A code spectrum can be considered as a "signature" of its associated network by which some characteristics can be estimated, including the refractory period and weight distribution. 
(5) A code can function as a mark on a spike wave. We have shown that to some extent, wave propagation preserves the codes. This finding was documented by movies as well as quantitatively.

Future studies will address the following:

(i) communication based on codes as a part of spike waves,

(ii) organization of communication links to derive intelligence functions.

\section{Conflict of Interests}

The authors declare that there is no conflict of interests regarding the publication of this paper.

\section{Acknowledgments}

This study was supported in part by the Grant-in-Aid for Scientific Research of Exploratory Research 21656100, 25630176, and Scientific Research (A) 22246054 of Japan Society for the Promotion of Science.

\section{References}

[1] B. Cessac, H. Paugam-Moisy, and T. Viéville, "Overview of facts and issues about neural coding by spikes," Journal of Physiology Paris, vol. 104, no. 1-2, pp. 5-18, 2010.

[2] O. Kliper, D. Horn, B. Quenet, and G. Dror, "Analysis of spatiotemporal patterns in a model of olfaction," Neurocomputing, vol. 58-60, pp. 1027-1032, 2004.

[3] K. Fujita, Y. Kashimori, and T. Kambara, "Spatiotemporal burst coding for extracting features of spatiotemporally varying stimuli," Biological Cybernetics, vol. 97, no. 4, pp. 293-305, 2007.

[4] I. Tyukin, T. Tyukina, and C. van Leeuwen, "Invariant template matching in systems with spatiotemporal coding: a matter of instability," Neural Networks, vol. 22, no. 4, pp. 425-449, 2009.

[5] A. Mohemmed, S. Schliebs, S. Matsuda, and N. Kasabov, "Training spiking neural networks to associate spatio-temporal input-output spike patterns," Neurocomputing, vol. 107, pp. 3-10, 2013.

[6] B. A. Olshausen and D. J. Field, "Emergence of simple-cell receptive field properties by learning a sparse code for natural images," Nature, vol. 381, no. 6583, pp. 607-609, 1996.

[7] A. J. Bell and T. J. Sejnowski, "The 'independent components' of natural scenes are edge filters," Vision Research, vol. 37, no. 23, pp. 3327-3338, 1997.

[8] S. Tamura, Y. Mizuno-Matsumoto, Y.-W. Chen, and K. Nakamura, "Association and abstraction on neural circuit loop and coding," in Proceedings of the 5th International Conference on Intelligent Information Hiding and Multimedia Signal Processing (IIHMSP '09), pp. 414-417, IEEE, Kyoto, Japan, September 2009.

[9] M. Abeles, Local Cortical Circuits: An Electrophysiological Study, Springer, Berlin, Germany, 1982.

[10] M. Abeles, "Synfire chains," Scholarpedia, vol. 4, no. 7, article 1441, 2009.

[11] E. M. Izhikevich, "Polychronization: computation with spikes," Neural Computation, vol. 18, no. 2, pp. 245-282, 2006.
[12] M. Perc, "Fluctuating excitability: a mechanism for selfsustained information flow in excitable arrays," Chaos, Solitons \& Fractals, vol. 32, no. 3, pp. 1118-1124, 2007.

[13] H. Zhang, Q. Wang, M. Perc, and G. Chen, "Synaptic plasticity induced transition of spike propagation in neuronal networks," Communications in Nonlinear Science and Numerical Simulation, vol. 18, no. 3, pp. 601-615, 2013.

[14] Y. Nishitani, C. Hosokawa, Y. Mizuno-Matsumoto, T. Miyoshi, H. Sawai, and S. Tamura, "Detection of M-sequences from spike sequence in neuronal networks," Computational Intelligence and Neuroscience, vol. 2012, Article ID 862579, 9 pages, 2012.

[15] P. L. Baljon, M. Chiappalone, and S. Martinoia, "Interaction of electrically evoked responses in networks of dissociated cortical neurons," Physical Review E, vol. 80, no. 3, Article ID 031906, 2009.

[16] S. Tamura, Y. Nishitani, C. Hosokawa et al., "Pseudo random sequences from neural circuits," in Proceedings of the International Forum on Medical Imaging in Asia (IFMIA '12), P. 144, Daejeon, Republic of Korea, November 2012, http://www .nbl-technovator.jp/NBL_Tech/paper/IFMIA48.pdf.

[17] T. Shinichi, Y. Nishitani, C. Hosokawa et al., "M-sequence family from cultured neural circuits," in Proceedings of the 3rd International Workshop on Computational Intelligence for BioMedical Science and Engineering (CIMSE '12), pp. 466-471, Taipei, Taiwan, October 2012, http://www.nbl-technovator.jp/ NBL_Tech/paper/MseqPSTH-Taipei3.pdf.

[18] R. C. Dixon, Spread Spectrum Systems, John Wiley \& Sons, 1976.

[19] D. V. Sarwate and M. B. Pursley, "Crosscorrelation properties of pseudorandom and related sequences," Proceedings of the IEEE, vol. 68, no. 5, pp. 593-619, 1980.

[20] S. W. Golomb and G. Gong, Signal Design for Good Correlation: for Wireless Communication, Ctyptography, and Rader, Cambridge University Press, Cambridge, UK, 2005.

[21] Y. M. Elyada and D. Horn, "Can dynamic neural filters produce pseudo-random sequences," in Artificial Neural Networks: Biological Inspiration-ICANN 2005, vol. 3696 of Lecture Notes in Computer Science, pp. 211-216, Springer, Heidelberg, Germany, 2005.

[22] S. Tamura, Y. Nishitani, C. Hosokawa et al., "Spike code flow in cultured neuronal networks," Computational Intelligence and Neuroscience, In press.

[23] T. Kamimura, Y. Yagi, S. Tamura, and Y.-W. Chen, "Multiplex communication with synchronous shift and weight learning in 2D mesh neural network," Automation, Control and Intelligent Systems, vol. 3, no. 5, pp. 63-70, 2015.

[24] Y. Mizuno-Matsumoto, K. Okazaki, A. Kato et al., "Visualization of epileptogenic phenomena using cross-correlation analysis: localization of epileptic foci and propagation of epileptiform discharges," IEEE Transactions on Biomedical Engineering, vol. 46, no. 3, pp. 271-279, 1999.

[25] W. Gerstner and W. M. Kistler, Spiking Neuron Models. Single Neurons, Populations, Plasticity, Cambridge University Press, 2002.

[26] D. J. Watts and S. H. Strogatz, "Collective dynamics of 'smallworld' networks," Nature, vol. 393, no. 6684, pp. 440-442, 1998.

[27] J. Koester and S. A. Siegelbaum, "Propagated signaling: the action potential," in Principles of Neural Science, E. Kandel, J. Schwartz, T. Jessell, S. Siegelbaum, and A. J. Hudspeth, Eds., chapter 7, p. 157, McGraw-Hill, New York, NY, USA, 5th edition, 2013. 
[28] S. Tamura, Y. Nishitani, T. Kamimura et al., "Multiplexed spatiotemporal communication model in artificial neural networks," Automation, Control and Intelligent Systems, vol. 1, no. 6, pp. 121-130, 2013.

[29] Y. Mizuno-Matsumoto, M. Ishijima, K. Shinosaki et al., "Transient global amnesia (TGA) in an MEG study," Brain Topography, vol. 13, no. 4, pp. 269-274, 2001.

[30] M. Perc, "Spatial coherence resonance in excitable media," Physical Review E, vol. 72, no. 1, pp. 1-6, 2005.

[31] K. Takahashi, S. Kim, T. P. Coleman et al., "Large-scale spatiotemporal spike patterning consistent with wave propagation in motor cortex," Nature Communications, vol. 6, article 7169, 2015.

[32] P. Spector, "Principles of cardiac electric propagation and their implications for re-entrant arrhythmias," Circulation: Arrhythmia and Electrophysiology, vol. 6, no. 3, pp. 655-661, 2013.

[33] P. A. Medolla, J. V. Arthur, R. Alvarez-Icaza et al., "A million spiking-neuron integrated circuit with a scalable communication network and interface," Science, vol. 345 , no. 6197, pp. 668673, 2014.

[34] S. Tamura, Y. Nishitani, C. Hosokawa, Y. Mizuno-Matsumoto, and Y.-W. Chen, "Base of brain intelligence: information flow in cultured neuronal networks and its simulation on 2D mesh network," in Proceedings of the 11th International Conference on Natural Computation and the 12th International Conference on Fuzzy Systems and Knowledge Discovery (ICNC-FSKD '15), IEEE Circuits and Systems Society, Zhangjiajie, China, August 2015, http://www.nbl-technovator.jp/NBL_Tech/paper/1551.pdf. 

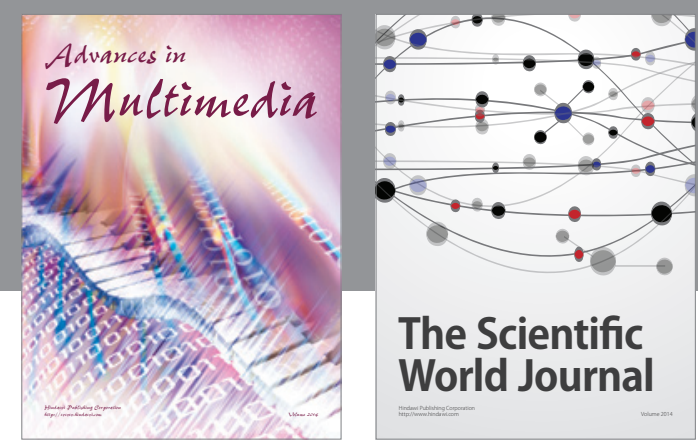

The Scientific World Journal
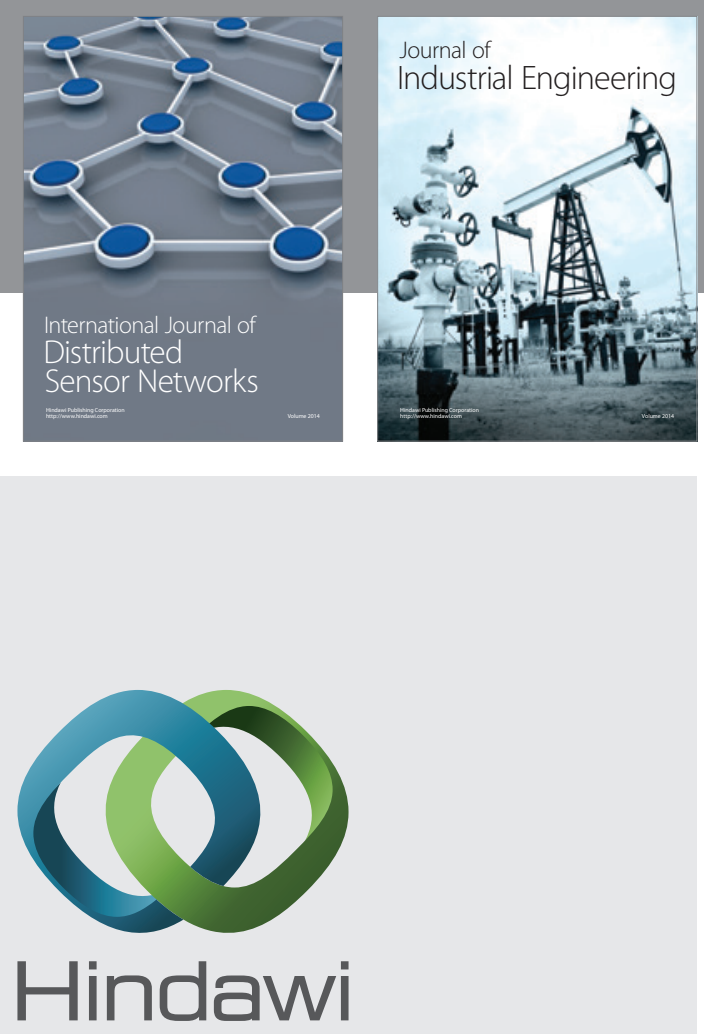

Submit your manuscripts at

http://www.hindawi.com

\section{Computer Networks} and Communications
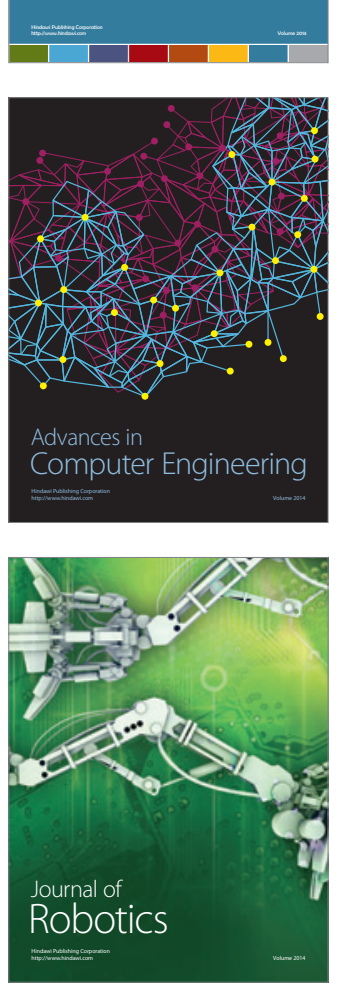
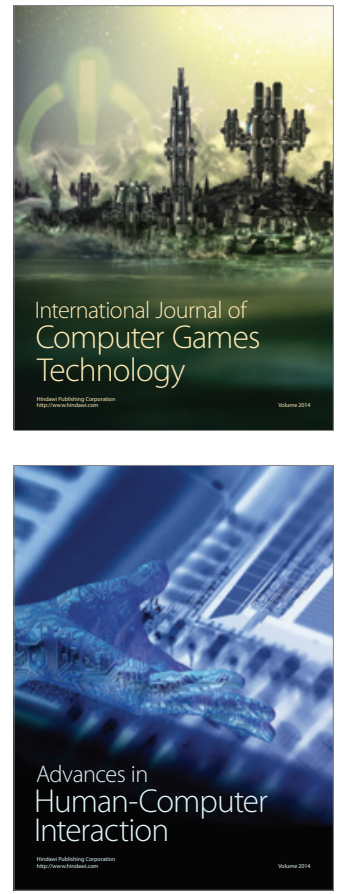
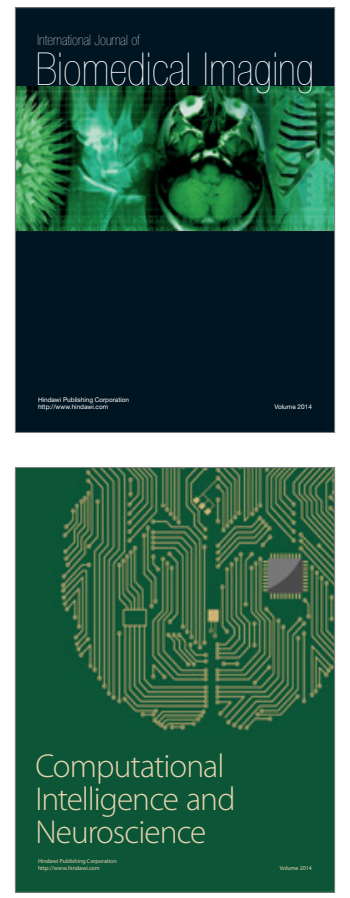
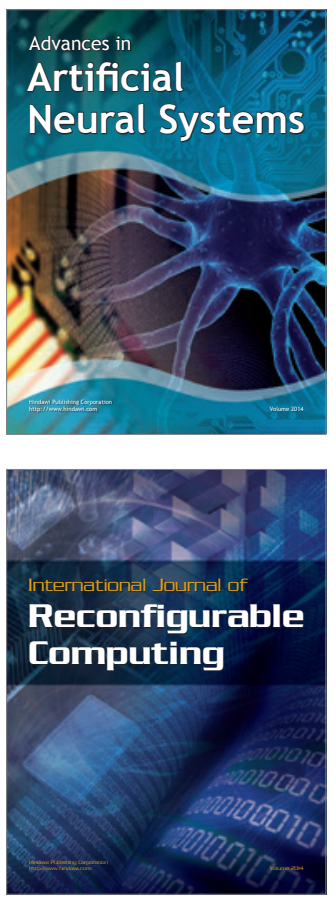
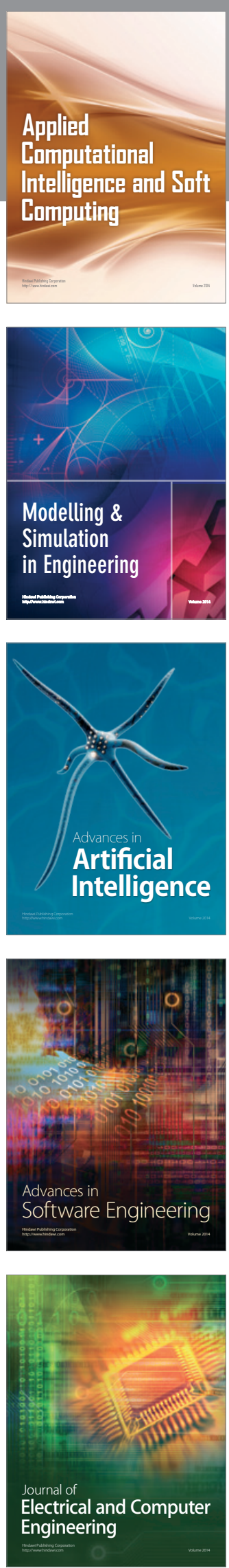